\title{
On the relation between motivation and retention in educational contexts: The role of intentional and unintentional mind wandering
}

\author{
Paul Seli $^{1}$ - Jeffrey D. Wammes ${ }^{2}$ • Evan F. Risko ${ }^{2}$ - Daniel Smilek ${ }^{2}$
}

Published online: 19 November 2015

(C) Psychonomic Society, Inc. 2015

\begin{abstract}
Highly motivated students often exhibit better academic performance than less motivated students. However, to date, the specific cognitive mechanisms through which motivation increases academic achievement are not well understood. Here we explored the possibility that mind wandering mediates the relation between motivation and academic performance, and additionally, we examined possible mediation by both intentional and unintentional forms of mind wandering. We found that participants reporting higher motivation to learn in a lecture-based setting tended to engage in less mind wandering, and that this decrease in mind wandering was in turn associated with greater retention of the lecture material. Critically, we also found that the influence of motivation on retention was mediated by both intentional and unintentional types of mind wandering. Not only do the present results advance our theoretical understanding of the mechanisms underlying the relation between motivation and academic achievement, they also provide insights into possible methods of intervention that may be useful in improving student retention in educational settings.
\end{abstract}

Keywords Mind wandering · Motivation · Intentional mind wandering $\cdot$ Unintentional mind wandering $\cdot$ Lectures

Electronic supplementary material The online version of this article (doi:10.3758/s13423-015-0979-0) contains supplementary material, which is available to authorized users.

Paul Seli

paulseli@fas.harvard.edu

1 Department of Psychology, Harvard University, William James Hall, 33 Kirkland Street, Cambridge, MA 02138, USA

2 Department of Psychology, University of Waterloo, Waterloo, Ontario, Canada
It has been well established that motivation is a key determinant of academic achievement, with more highly motivated students typically outperforming their less motivated colleagues (e.g., Pintrich, 1999; Schiefele, Krapp, \& Winteler, 1992). However, to date, the specific cognitive mechanism(s) through which motivation influences academic achievement are not well understood, and only recently has research begun to systematically explore such mechanisms. In one such study, it was suggested that the relation between motivation and academic performance is mediated by mind wandering: specifically, that poorly motivated students frequently engage in mind wandering, which in turn results in poorer retention (Unsworth \& McMillan, 2013). Here we further refine this theoretical proposal by examining whether the relation between motivation and retention might be differentially associated with intentional and unintentional forms of mind wandering. In addition, we expand previous research on the relations among motivation, mind wandering, and retention by examining the effects of these variables in a novel educational context - namely, during a lecture. Thus, the present investigation both advances our theoretical understanding of the mechanism(s) underlying the relation between motivation and academic achievement and generalizes this research to a novel educational context.

In recent work, Unsworth and McMillan (2013) explored the effects of motivation and mind wandering on reading comprehension in a laboratory setting. To this end, the researchers had participants read part of a chapter from a textbook, and while reading, the participants were occasionally presented with thought probes to assess whether they were "mind wandering" or "focused on the task." Following the reading task, participants were tested on the textbook material and were then presented two questions assessing their motivation to do well on the reading comprehension test. Critically, the researchers found evidence that the well-established relation between motivation and task performance (in this case, 
reading comprehension; e.g., Humphreys \& Revelle, 1984; Pintrich \& De Groot, 1990) was fully mediated by mindwandering rates: Participants who reported lower levels of motivation were more likely to engage in mind wandering during the reading task, and this increased propensity to engage in mind wandering negatively predicted test performance.

Although Unsworth and McMillan (2013) provided evidence consistent with the claim that mind wandering mediates the relation between motivation and retention, it is important to note that a common assumption in the literature on mind wandering is that participants' reports of "mind wandering" reflect unintentionally occurring off-task thoughts (e.g., Kane \& McVay, 2012; McVay \& Kane, 2010; Seli, Cheyne, \& Smilek, 2013; Seli, Carriere, Thomson, Cheyne, Martens, \& Smilek 2014; Smallwood \& Schooler, 2006; see the supplementary materials for quotations from these and numerous other authors who have either explicitly stated or clearly implied that mind wandering occurs spontaneously/without intention). Thus, in the context of Unsworth and McMillan's study, one might assume that the findings indicate that unintentional mind wandering mediates the relationship between motivation and retention. However, mounting evidence suggests that participants" reports of "mind wandering" do not exclusively reflect unintentionally engaged mind wandering, but also frequently reflect deliberately engaged mind wandering (e.g., Carriere, Seli, \& Smilek, 2013; Forster \& Lavie, 2009; Phillips, Mills, D’Mello, \& Risko, 2015; Seli, Cheyne, Xu, Purdon, \& Smilek, 2015b; Seli, Smallwood, Cheyne, \& Smilek, 2015). Importantly, research examining intentional and unintentional mind wandering has suggested that these two types of mind wandering are dissociable, being differentially affected by task demands such as rereading text (Phillips et al., 2015) and variations in task difficulty (Seli, Risko, \& Smilek, 2015), and that they are also differentially related to other constructs, such as dimensions of mindfulness (Seli, Carriere, \& Smilek, 2015a). Thus, given that Unsworth and McMillan did not distinguish between these two types of mind wandering, it is possible that intentional mind wandering is partially, or possibly even fully, responsible for the observed mediation of the relation between motivation and retention.

Further reason to suspect that intentional mind wandering might account for the relation between motivation and comprehension comes from consideration of the possible associations between the two types of mind wandering and motivation. In particular, it seems reasonable to assume that motivation ought to be strongly related to intentional mind wandering. Indeed, individuals reporting low levels of task-based motivation should seemingly be more likely to intentionally disengage from their task in the service of focusing on internally generated thought. On the other hand, motivation might be less likely to be associated with one's propensity to unintentionally engage in mind wandering, since such mental experiences are, by definition, not under the participant's control. In support of these predictions, Seli, Cheyne, et al. (2015) recently observed a negative correlation between participants' motivation and their rates of intentional mind wandering during a sustained-attention task, but found no relation between motivation and rates of unintentional mind wandering. Following from these results, a strong prediction is that, rather than unintentional mind wandering, intentional mind wandering could be responsible for the relation between motivation and comprehension.

Although there seems to be good reason to suspect that intentional mind wandering might exclusively mediate the relation between motivation and comprehension, it is of course possible that both intentional and unintentional mind wandering play mediating roles. Whereas Seli, Cheyne, et al. (2015) did not find a significant association between unintentional mind wandering and motivation, their study involved a sustained-attention task, which may produce very different results than tasks assessing comprehension/retention. Moreover, Seli, Cheyne, et al. (2015) reported a negative, albeit nonsignificant, correlation between motivation and unintentional mind wandering, which suggests the possibility that these variables may be weakly related to one another.

In summary, three theoretically interesting patterns of results exist that could characterize the relation between motivation, mind wandering, and comprehension. The first is that unintentional, but not intentional, mind wandering mediates the relation between motivation and performance. This is arguably the view that most mind-wandering researchers would endorse, given the Unsworth and McMillan (2013) results. The second, which is predicted on the basis of the aforementioned work examining motivation and the intentionality of mind wandering (Seli, Cheyne, et al., 2015), is that intentional, but not unintentional, mind wandering mediates the relation. The third and final possible pattern of results is that the influence of motivation on retention is mediated by both intentional and unintentional mind wandering. It is important to note that, given the assumption that mind wandering is largely unintentional, Pattern 2 or Pattern 3 would represent an important advancement in our understanding of the relation between motivation and academic performance (i.e., either of these findings would suggest that intentional mind wandering plays an important role in the relation between motivation and academic performance).

Determining the specific relations among intentional and unintentional mind wandering with motivation and comprehension is important, because the type of mind wandering that people engage in has important implications for understanding the mechanisms involved in these episodes of off-task thought. Moreover, determining whether these two types of mind wandering are uniquely related to motivation and test performance could also have important implications for interventions designed to improve learning. If, for example, mind wandering in educational settings is indeed primarily unintentional, then the focus of interventions might be on modifying the ways in 
which material is presented, such that the delivery of the material is continually salient and exogenously draws attention. For instance, the focus might be on increasing the use of multimedia in lectures (Lenzner, Schnotz, \& Müller, 2013). If, however, at least some of the mind wandering that occurs in educational settings is engaged with intention, this finding would suggest that researchers and pedagogical practitioners should also focus on methods of intervention aimed at reducing intentional, controlled mind wandering (e.g., by increasing incentives to focus on the lecture rather than intentionally disengage from it).

\section{The present study}

Although understanding the relations among motivation, mind wandering, and performance is of general theoretical interest, understanding how these variables interact in lecture settings is particularly important. In Unsworth and McMillan (2013), the researchers focused specifically on reading comprehension. Here we extend this work to the lecture setting, which is one of the most common pedagogical formats. In a series of recent articles, researchers have begun to better understand the relation between mind wandering and the retention of lecture material (e.g., Farley, Risko, \& Kingstone, 2013; Risko, Anderson, Sarwal, Engelhardt, \& Kingstone, 2012; Risko, Buchanan, Medimorec, \& Kingstone, 2013; Szpunar, Khan, \& Schacter, 2013). As in research examining reading comprehension, this work has revealed that mind wandering is negatively associated with the retention of lecture material. Critically, however, no previous work has investigated the relation between motivation, mind wandering, and retention in lectures, nor has any research investigated different forms of mind wandering (i.e., intentional vs. unintentional) in the lecture context.

In the present study, participants viewed a video-recorded lecture, and at various points throughout the lecture, we presented thought probes that were used to identify periods of intentional mind wandering, unintentional mind wandering, and/or on-task focus. Following the lecture, we tested participants on their retention of the lecture material, after which they reported how motivated they had been to perform well on the task. A series of correlation and mediation analyses were performed to assess predictions with respect to the relation between overall, intentional, and unintentional mind wandering, motivation, and lecture retention.

\section{Method}

\section{Participants}

A total of 120 undergraduate students enrolled in psychology courses at the University of Waterloo participated in exchange for partial course credit. We determined in advance that we would stop data collection at 120 participants, because this sample size is appropriate for the mediation analyses we sought to conduct.

\section{Stimuli}

Participants viewed a video recording of a live lecture given in a lecture hall (the video was obtained from Open Yale Courses; http://oyc.yale.edu/). The lecture was roughly $25 \mathrm{~min}$ in length and focused on mortality decline in Europe from the 1500 s to the 1900 s.

\section{Thought probes}

Throughout the lecture, one thought probe was presented at a random interval in each of a succeeding series of 90-s blocks (16 probes). Upon the presentation of each probe, the lecture was paused until a response had been given. The probes asked participants to indicate whether they had just been (1) focused on the task, (2) intentionally mind wandering, and/or (3) unintentionally mind wandering. Participants were allowed to select just one response, if that was appropriate (e.g., intentionally mind wandering), or to select any combination of responses (e.g., intentionally and unintentionally mind wandering) by using the mouse to click boxes placed beside each of the three response options. We used this method rather than forcing participants to choose only one response because, in the case of the forced choice methodology, there is a structurally forced negative correlation of the different report types (i.e., the responses are partially ipsative), which precludes analyses examining the independent contributions of these different report types. ${ }^{1}$

\section{Retention of lecture material}

Following the lecture, participants were presented with nine multiple-choice questions about the content of the lecture. These were presented one at a time, in a randomized order. Participants were given as much time as needed to respond to each question, and after they had provided a response, the next question appeared. Each question had four possible response options, only one of which was correct.

\section{Motivation}

Following the retention test, participants were presented a single-item question asking them "How motivated were you to perform well on the task?" (Unsworth \& McMillan, 2013). The anchor ratings for this question were 1 (not motivated at all) and 7 (very motivated).

\footnotetext{
${ }^{1}$ For a discussion of the potentially problematic role of ipsative data in mind-wandering research, see the supplementary materials.
} 


\section{Procedure}

Participants were seated in front of a computer monitor. They were instructed to attend to the lecture and to do their best to retain the material presented, because they would be tested on this material. They were also told that, throughout the lecture, they would be presented with probes asking them to report whether they were on task, intentionally mind wandering, unintentionally mind wandering, or any combination of these three reports. Following the lecture, the retention test was administered, followed by a single-item question asking about the participant's motivation.

\section{Measures}

For each participant, we computed three thought probe measures. These included the proportions of (1) on-task reports, (2) intentional mind-wandering reports, and (3) unintentional mind-wandering reports. In addition, we computed each participant's mean accuracy on the retention test by averaging their scores ( 1 for correct and 0 for incorrect) across the nine multiple-choice questions. Finally, our measure of task-based motivation was the response to the single-item motivation question.

\section{Results}

We present descriptive statistics for all measures in Table 1. As can be seen in the table, the skewness and kurtosis values for the proportion of intentional mind wandering were beyond an acceptable range (i.e., skewness $>2$ and kurtosis $>4$; Kline, 1998). We therefore transformed these data using a squareroot transformation, which brought the skewness and kurtosis values into an acceptable range (post-transformation skewness $=0.46$, post-transformation kurtosis $=-0.73){ }^{2}$ In further examining Table 1, it is worth noting is that participants correctly responded to $63 \%$ of the retention-test questions, which was significantly higher than chance performance $(25 \%), t(119)=$ $19.48, S E=0.38, p<.001$.

Next, we examined the Pearson product-moment correlation coefficients for all primary measures. In addition, we were interested in exploring how overall mind wandering (i.e., the proportion of reports of intentional mind wandering, unintentional mind wandering, and concurrent experiences of intentional and unintentional mind wandering) related to our primary measures. To clarify, any time a participant reported any form of mind wandering - be it intentional, unintentional, or a combination of both - this contributed to the measure of

\footnotetext{
${ }^{2}$ In all subsequent analyses examining intentional mind wandering, the transformed data with corrected skewness and kurtosis levels were used.
}

Table 1 Psychometric properties of all primary measures $(N=120)$

\begin{tabular}{llll}
\hline & Mean (SD) & Skewness $^{1}$ & Kurtosis $^{2}$ \\
\hline On task & $.737(.21)$ & -0.90 & 0.79 \\
Intentional mind wandering & $.088(.12)$ & 2.07 & 6.00 \\
Unintentional mind wandering & $.239(.18)$ & 0.87 & 1.47 \\
Motivation & $4.73(1.35)$ & -0.58 & 0.90 \\
Retention & $.632(.22)$ & -0.35 & -0.69 \\
\hline
\end{tabular}

${ }^{1}$ Std. error $=.221,{ }^{2}$ Std. error $=.438$

overall mind wandering. The results of the correlation analysis are presented in Table 2.

Consistent with previous research (Seli, Cheyne, et al., 2015), we observed a significant positive correlation between the proportion of on-task reports and motivation, indicating that individuals who reported higher motivation to perform well on the task more frequently reported being focused on the task. We also observed a positive correlation between the proportion of on-task reports and accuracy on the retention test: Individuals reporting more periods of on-task focus were more likely to perform well on the retention test. Also of interest was the finding that both intentional and unintentional mind wandering were negatively associated with accuracy on the retention test, as was overall mind wandering. Finally, we found that motivation was negatively associated with each type of mind wandering (intentional and unintentional), as well as with overall mind wandering, and in addition, we observed a positive correlation between motivation and accuracy on the retention test.

Taken together, these results suggest the possibility that the association of motivation and task performance may be, as was reported by Unsworth and McMillan (2013), mediated by mind wandering. Thus, in our next analysis, we formally tested this possibility by conducting a mediation analysis. To estimate indirect effects in our mediation models, we used the PROCESS modeling tool (Hayes, 2012), set to Model 4, with 1,000 bootstrap samples, and a $95 \%$ confidence level for the confidence intervals (CIs). The mediation model with unstandardized regression coefficients is depicted in Fig. 1. Critically, the indirect effect, .0153 (95\% CI: .0052-.0338), was significant (i.e., the $95 \%$ CI did not include 0), suggesting that the influence of motivation on task performance is mediated by mind wandering.

Consistent with Unsworth and McMillan's (2013) prior work, the results of the mediation analysis indicated that mind wandering is a mechanism through which motivation influences the retention of lecture material. Specifically, individuals reporting higher levels of motivation tend to engage in mind wandering less frequently, which in turn is associated with improved retention relative to individuals reporting low motivation. 
Table 2 Pearson productmoment correlations of all primary measures as well as overall mind wandering $(N=120)$

\begin{tabular}{|c|c|c|c|c|c|}
\hline & 2 & 3 & 4 & 5 & 6 \\
\hline 1. On task & $-.57^{* * *}$ & $-.69^{* * *}$ & $-.81^{* * *}$ & $.31^{* * *}$ & $.44^{* * *}$ \\
\hline 2. Intentional mind wandering & - & $.30^{* * *}$ & $.68^{* * *}$ & $-.26^{* *}$ & $-.27^{* *}$ \\
\hline 3. Unintentional mind wandering & & - & $.88^{* * *}$ & $-.23^{*}$ & $-.30^{* * *}$ \\
\hline 4. Overall mind wandering & & & - & $-.29^{* * *}$ & $-.36^{* * *}$ \\
\hline 5. Motivation & & & & - & $.18^{*}$ \\
\hline 6. Retention & & & & & - \\
\hline
\end{tabular}

Having found that the relation of motivation and retention is mediated by overall mind-wandering rates, we next wanted to examine whether the relation between motivation and retention is mediated by intentional mind wandering, unintentional mind wandering, or both. Intentional and unintentional mind wandering were significantly correlated with one another, but we did not predict this to be a causal relation. Accordingly, rather than house both variables in the same mediation model, separate models were tested for each mind-wandering response. To be conservative in our analyses, when testing for indirect effects through one type of mind wandering, the other type of mind-wandering response was included in the model as a covariate, acting on both the mediator and the outcome variable (retention).

We first turned our attention to intentional mind wandering. The PROCESS modeling tool (Hayes, 2012) was used to estimate the indirect effect through intentional mind wandering (controlling for unintentional mind wandering). PROCESS was set to Model 4, with 1,000 bootstrap samples, and a $95 \%$ confidence level for the CIs. The mediation model with unstandardized regression coefficients is depicted in Fig. 2. The indirect effect through intentional mind wandering was significant, .0060 (95 \% CI: .0005-.0171) (Fig. 2).

The indirect effect through unintentional mind wandering was analyzed in the same manner, with intentional mind

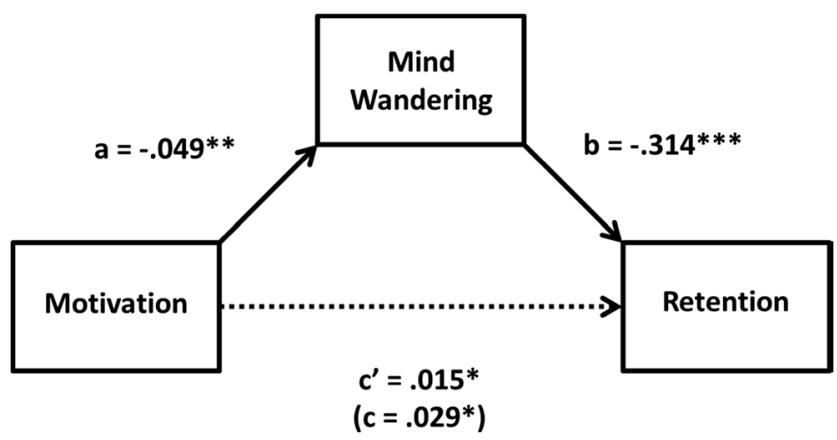

Fig. 1 PROCESS mediation model depicting the relationship between self-reported motivation and the retention of lecture material, with the overall mind-wandering rate as a mediator. The indirect effect of motivation on retention through mind wandering is reported as $c^{\prime}$, and the total effect of motivation on retention is in parentheses below $(c)$. The significance of the coefficients is represented using the following notation: ${ }^{*} p<.05,{ }^{* *} p<.01,{ }^{* * *} p<.001$ wandering included as a covariate. Note that the direct effect of motivation on unintentional mind wandering was marginal $(p=.077)$. However, the indirect effect through unintentional mind wandering, .0058 (95 \% CI: .0003-.0218), was nevertheless significant (i.e., the $95 \% \mathrm{CI}$ did not include 0 ; see Fig. 3). In both models the direct effect was not significant, $p=.368$, consistent with the notion that the effect of motivation on later retention of lecture material was mediated by both unintentional and intentional mind wandering.

\section{Discussion}

Here we examined a recent proposal that motivation-triggered shifts of attention toward educational material may be a key mechanism through which motivation acts on academic performance. Consistent with this theoretical view, we found that individuals reporting higher motivation to learn in a lecturebased setting tended to engage in less mind wandering, and this decrease in mind wandering was in turn associated with greater retention of the lecture material. Critically, we also found that the influence of motivation on retention is mediated by both intentional and unintentional mind wandering:

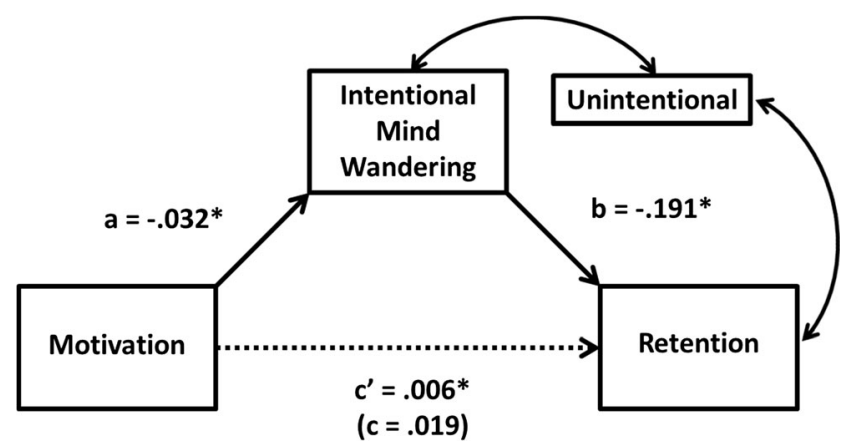

Fig. 2 PROCESS mediation model depicting the relationship between self-reported motivation and the retention of lecture material, with the intentional mind-wandering rate as a mediator and unintentional mind wandering as a covariate on both the mediator (intentional mind wandering) and the outcome variable (retention). The indirect effect of motivation on retention through intentional mind wandering is reported as $c^{\prime}$, and the total effect of motivation on retention is in parentheses below $(c)$. The significance of the coefficients is represented using the following notation: ${ }^{*} p<.05$ 


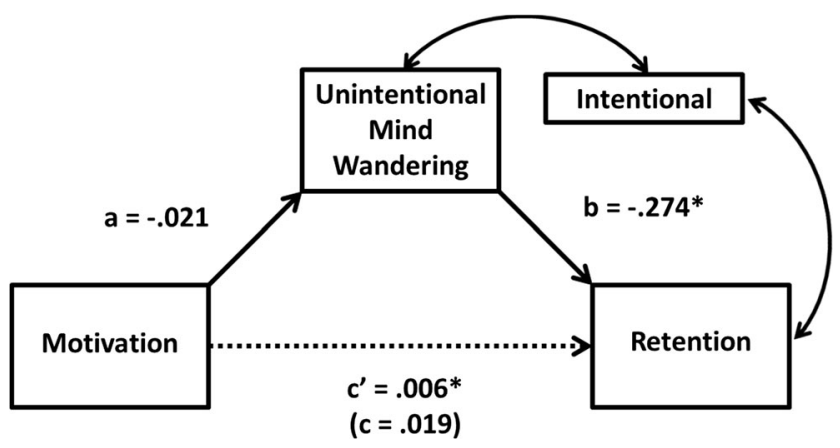

Fig. 3 PROCESS mediation model depicting the relationship between self-reported motivation and the retention of lecture material, with the unintentional mind-wandering rate as a mediator and intentional mind wandering as a covariate on both the mediator (unintentional mind wandering) and the outcome variable (retention). The indirect effect of motivation on retention through unintentional mind wandering is reported as $c^{\prime}$, and the total effect of motivation on retention is in parentheses below $(c)$. The significance of the coefficients is represented using the following notation: ${ }^{*} p<.05$

Participants who reported higher levels of motivation tended to engage in less of both intentional and unintentional mind wandering, and reductions in each of these types of mind wandering were associated with improved retention. Thus, the proposed link between motivation and retention through mind wandering follows two independent paths: one through intentional mind wandering, and another through unintentional mind wandering.

The finding that unintentional mind wandering mediated the relation between motivation and retention is intriguing and worth consideration. At first blush, it is not obvious why motivation would be related to unintentional bouts of mind wandering. However, upon consideration of this result, we reasoned that this relation might exist for several reasons. One possibility is that people who are highly motivated to do well might also be more motivated to catch themselves mind wandering and to terminate this process. Indirect support for this view has come from studies showing that increases in motivation are associated with increased sensitivity of the anterior cingulate cortex (see Sarter, Gehring, \& Kozak, 2006), a brain area that is believed to play an important role in conflict detection (e.g., Botvinick, Cohen, \& Carter, 2004; Brown \& Braver, 2005; Gehring \& Taylor, 2004). This is consistent with the possibility that increases in task-based motivation would be associated with an increase in the detection of episodes of mind wandering (reflecting a conflict between one's goals and one's behaviors/cognitions), which should in turn be associated with an increased propensity to self-catch and terminate bouts of mind wandering. A second possibility is that high levels of motivation buffer individuals from otherwise intrusive, unintentional mental activity. Some support for this view has come from Pessoa (2009), who suggested that increases in motivation lead to improvements in the efficiency with which individuals can orient their attention. Indeed,
Pessoa argued that motivation can serve to "recalibrate the allocation of processing resources available to executive functions" (p. 164), and further, that such reallocation should "impact not only target functions directly associated with rewarded behaviors, but other processes that share some of the same processing resources" (p. 164). According to this view, then, participants who are highly motivated to attend to a given task should be better able to allocate their processing resources to the task, which should be associated with a reduction in the allocation of resources to task-irrelevant thoughts (or mind wandering). At present, however, it is unclear whether either or both of these accounts are responsible for the observed relation between motivation and unintentional mind wandering. Thus, future work exploring these (and other) possibilities will likely yield further insights into these important relations.

\section{Limitations of the present work}

One potential limitation of the present study (and of Unsworth \& McMillan's, 2013, study) that is worth noting is that, in both of these studies, participants' motivation was assessed at the end of the experiment. Thus, both here and in Unsworth and McMillan's study, it is possible that participants' motivation reports were influenced by their rates of mind wandering and/ or their performance on the comprehension test. In particular, the possibility exists that (1) participants reporting high rates of mind wandering may consequently have been inclined to report lower motivation, and (2) participants who performed poorly on the retention test may have similarly felt inclined to report low levels of task-based motivation, even if they in fact had been highly motivated to perform well. Below, we consider these possibilities separately.

In considering the first possibility (that mind-wandering rates bias motivation reports), it is worth noting that how the direction of causality between mind wandering and motivation might be addressed via self-reports is not clear. One suggestion might be to assess motivation at the beginning (rather than at the end) of the study. However, in the same way that rates of mind wandering might produce biased reports of motivation when participant motivation is assessed at the end of the experiment, reporting one's level of motivation at the beginning might likewise bias subsequently obtained reports to thought probes. In addition to this, in many experimental designs it is not clear how participants would even begin to confidently report on their task-based motivation without having first experienced the task. In the case of the present study, to provide an accurate report of task-based motivation at the beginning of the experiment, the participant would presumably require information about the content of the lecture, the style of the lecturer, the visual aids used, et cetera, thus making this procedure difficult to implement. One possible method for future research might be to employ experimental 
manipulations of task interest or incentives to affect motivation. In any case, it seems likely that the covariation of motivation and mind wandering might well be bidirectional.

With respect to the second possibility (that performance on the retention test may have biased motivation reports), at least two considerations dampen this concern. The first is that, in the present study, each question from the retention test was a multiple-choice question with four possible responses, and participants were not provided feedback. As such, participants likely did not know how well they had performed, in which case, performance could not have influenced motivation reports . Second, in the case that test performance was inadvertently influencing participants' reports of motivation, one might expect to observe a stronger correlation between motivation and test performance than was observed here. For these reasons, then, we feel that concerns regarding the assessment of motivation in the present study are minimized. That being said, we believe that consideration of the complications surrounding assessments of motivation will be important for future research.

\section{Theoretical and practical implications}

One important theoretical implication from the present study is that it may be difficult (if not impossible) to distinguish between intentional and unintentional mind wandering on the basis of performance alone. In the present study, although test retention was uniquely associated with rates of intentional and unintentional mind wandering, it was not differentially related to these two types of mind wandering: Increases in both types of mind wandering were associated with poorer test performance. This finding makes good sense. Indeed, by definition, both intentional and unintentional mind wandering involve the withdrawal of attention from one's primary task, and both should be expected to be associated with similar (or identical) performance decrements. However, despite the fact that both types of mind wandering may have indistinguishable influences on performance across numerous tasks, it is of critical importance that researchers distinguish between these two types of mind wandering. Indeed, whether one intentionally or unintentionally engages in mind wandering has major implications for the underlying mechanisms involved in these episodes, even if the performance costs are identical.

It is important to note that, whereas in the present experiment unintentional and intentional mind wandering had similar effects on performance, and were also similar in terms of mediating the relation between motivation and performance, these types of mind wandering do not always behave in this manner (i.e., they are dissociable). For example, Shaw and Giambra (1993) found that, whereas individuals diagnosed with ADHD report more bouts of unintentional mind wandering in laboratory settings, they show no difference in rates of intentional mind wandering relative to healthy controls (see also Seli, Smallwood, et al., 2015). In addition, Phillips, Mills,
D'Mello, and Risko (2015) recently demonstrated that, although rereading a section of text was associated with increases in overall mind wandering, this increase was attributable to increases in intentional, but not unintentional, mind wandering. Moreover, research has shown that, whereas intentional mind wandering is positively associated with nonreactivity to one's inner experiences (a facet of mindfulness), unintentional mind wandering is negatively associated with this variable (Seli et al., 2015a). Finally, in an examination of the relations of state-level (in-laboratory) and trait-level (everyday-life) reports of intentional and unintentional mind wandering, Seli, Risko, and Smilek (2015) showed a correspondence between trait-level reports and their state-level counterparts: In particular, in-laboratory reports of intentional mind wandering were positively associated with trait-level reports of intentional, but not unintentional, mind wandering, and conversely, in-laboratory reports of unintentional mind wandering were positively associated with trait-level reports of unintentional, but not intentional, mind wandering. Taken together, these results are particularly important, because it might be argued that, in the present study, no evidence suggests unique theoretical underpinnings of intentional and unintentional types of mind wandering, given that they had similar associations with motivation and retention. However, the foregoing demonstrations that intentional and unintentional mind wandering are dissociable in numerous other contexts effectively addresses this concern.

In addition to having important theoretical implications, the present results are of particular importance for pedagogical purposes. Although efforts to minimize "mind wandering" have specifically focused on reducing the occurrence of unintentional task-unrelated thoughts (e.g., Mrazek, Smallwood, \& Schooler, 2012), the results of our study suggest that attempts to reduce intentional task-unrelated thoughts should also improve students' retention of educational material. One possible way to reduce intentional mind wandering may be to frequently administer tests during lectures. Indeed, recent work (Szpunar et al., 2013) has demonstrated that intermittent testing during lectures reduces mind wandering and improves learning, possibly because the testing episode provides feedback that helps counteract a student's typical overconfidence in his or her learning (Szpunar, Jing, \& Schacter, 2014). Since students who are overconfident in their knowledge of the lecture material might be particularly inclined to intentionally disengage from the lecture, the correction in confidence that comes with testing might have the impact of reducing intentional mind wandering. Another potentially effective way to reduce intentional mind wandering might be simply to inform students about the deleterious consequences of intentional mind wandering and to encourage them to avoid disengaging intentionally. After all, by definition, intentional mind wandering is under one's control. 
Author note This research was supported by a Natural Sciences and Engineering Research Council of Canada (NSERC) Discovery Grant to D.S. and E.F.R., by funding from the Canada Research Chairs program for E.F.R., and by an NSERC Postdoctoral Fellowship to P.S.

\section{References}

Botvinick, M. M., Cohen, J. D., \& Carter, C. S. (2004). Conflict monitoring and anterior cingulate cortex: An update. Trends in Cognitive Sciences, 8, 539-546. doi:10.1016/j.tics.2004.10.003

Brown, J. W., \& Braver, T. S. (2005). Learned predictions of error likelihood in the anterior cingulate cortex. Science, 307, 1118-1121. doi:10.1126/science. 1105783

Carriere, J. S. A., Seli, P., \& Smilek, D. (2013). Wandering in both mind and body: Individual differences in mind wandering and inattention predict fidgeting. Canadian Journal of Experimental Psychology, 67, 19-31. doi:10.1037/a0031438

Farley, J., Risko, E. F., \& Kingstone, A. (2013). Everyday attention and lecture retention: The effects of time, fidgeting, and mind wandering. Frontiers in Psychology, 4, 619. doi:10.3389/fpsyg.2013.00619

Forster, S., \& Lavie, N. (2009). Harnessing the wandering mind: The role of perceptual load. Cognition, 111, 345-355. doi:10.1016/j. cognition.2009.02.006

Gehring, W. J., \& Taylor, S. F. (2004). When the going gets tough, the cingulate gets going. Nature Neuroscience, 7, 1285-1287.

Hayes, A. F. (2012). PROCESS: A versatile computational tool for observed variable mediation, moderation, and conditional process modeling. Retrieved from www.afhayes.com/public/process 2012 . pdf

Humphreys, M. S., \& Revelle, W. (1984). Personality, motivation, and performance: A theory of the relationship between individual differences and information processing. Psychological Review, 91, 153184. doi:10.1037/0033-295X.91.2.153

Kane, M. J., \& McVay, J. C. (2012). What mind wandering reveals about executive-control abilities and failures. Current Directions in Psychological Science, 21, 348-354. doi:10.1177/ 0963721412454875

Kline, R. B. (1998). Principles and practice of structural equation modeling. New York: Guilford Press.

Lenzner, A., Schnotz, W., \& Müller, A. (2013). The role of decorative pictures in learning. Instructional Science, 41, 811-831.

McVay, J. C., \& Kane, M. J. (2010). Does mind wandering reflect executive function or executive failure? Comment on Smallwood and Schooler (2006) and Watkins (2008). Psychological Bulletin, 136, 188-197. doi:10.1037/a0018298. disc. 198-207.

Mrazek, M. D., Smallwood, J., \& Schooler, J. W. (2012). Mindfulness and mind-wandering: Finding convergence through opposing constructs. Emotion, 12, 442-448. doi:10.1037/a0026678

Pessoa, L. (2009). How do emotion and motivation direct executive control? Trends in Cognitive Sciences, 13, 160-166. doi:10.1016/j.tics. 2009.01.006

Phillips, N., Mills, C., D’Mello, S., \& Risko, E. F. (2015). On the influence of re-reading on mind wandering. Quarterly Journal of Experimental Psychology.

Pintrich, P. R. (1999). The role of motivation in promoting and sustaining self-regulated learning. International Journal of Educational Research, 31, 459-470.
Pintrich, P. R., \& De Groot, E. V. (1990). Motivational and self-regulated learning components of classroom academic performance. Journal of Educational Psychology, 82, 33-40.

Risko, E. F., Anderson, N., Sarwal, A., Engelhardt, M., \& Kingstone, A. (2012). Everyday attention: Variation in mind wandering and memory in a lecture. Applied Cognitive Psychology, 26, 234-242. doi:10. 1002/acp.1814

Risko, E. F., Buchanan, D., Medimorec, S., \& Kingstone, A. (2013). Everyday attention: Mind wandering and computer use during lectures. Computers and Education, 68, 275-283.

Sarter, M., Gehring, W. J., \& Kozak, R. (2006). More attention must be paid: The neurobiology of attentional effort. Brain Research Reviews, 51, 145-160.

Schiefele, J., Krapp, A., \& Winteler, A. (1992). Interest as a predictor of academic achievement: A meta-analysis of research. In K. A. Renninger, S. Hidi, \& A. Krapp (Eds.), The role of interest in learning and development. Hillsdale: Erlbaum.

Seli, P., Carriere, J. S. A., Thomson, D. R., Cheyne, J. A., Martens, K. A. E., \& Smilek, D. (2014). Restless mind, restless body. Journal of Experimental Psychology: Learning, Memory, and Cognition, 40, 660-668.

Seli, P., Carriere, J. S. A., \& Smilek, D. (2015a). Not all mind wandering is created equal: Dissociating deliberate from spontaneous mind wandering. Psychological Research, 79, 750-758. doi:10.1007/ s00426-014-0617-x

Seli, P., Cheyne, J. A., Xu, M., Purdon, C., \& Smilek, D. (2015b). Motivation, intentionality, and mind wandering: Implications for assessments of task-unrelated thought. Journal of Experimental Psychology: Learning, Memory, and Cognition, 41, 1417-1425. doi:10.1037/xlm0000116

Seli, P., Cheyne, J. A., \& Smilek, D. (2013). Wandering minds and wavering rhythms: Linking mind wandering and behavioral variability. Journal of Experimental Psychology: Human Perception and Performance, 39, 1-5. doi:10.1037/a0030954

Seli, P., Risko, E. F., \& Smilek, D. (2015). On the necessity of distinguishing between unintentional and intentional mindwandering. Manuscript under review.

Seli, P., Smallwood, J., Cheyne, J. A., \& Smilek, D. (2015d). On the relation of mind wandering and ADHD symptomatology. Psychonomic Bulletin \& Review, 22, 629-636. doi:10.3758/ s13423-014-0793-0

Shaw, G. A., \& Giambra, L. M. (1993). Task-unrelated thoughts of college students diagnosed as hyperactive in childhood. Developmental Neuropsychology, 9, 17-30. doi:10.1080/87565649309540541

Smallwood, J., \& Schooler, J. W. (2006). The restless mind. Psychological Bulletin, 132, 946-958. doi:10.1037/0033-2909. 132.6.946

Szpunar, K. K., Jing, H. G., \& Schacter, D. L. (2014). Overcoming overconfidence in learning from video-recorded lectures: Implications of interpolated testing for online education. Journal of Applied Research in Memory and Cognition, 3, 161-164.

Szpunar, K. K., Khan, N. Y., \& Schacter, D. L. (2013). Interpolated memory tests reduce mind wandering and improve learning of online lectures. Proceedings of the National Academy of Sciences, 110, 6313-6317. doi:10.1073/pnas. 1221764110

Unsworth, N., \& McMillan, B. D. (2013). Mind wandering and reading comprehension: Examining the roles of working memory capacity, interest, motivation, and topic experience. Journal of Experimental Psychology: Learning, Memory, and Cognition, 39, 832-842. doi: 10.1037/a0029669 